\title{
Psychology
}

\section{GW23-e2187 ONE CASE REPORT OF DEPRESSION CHARACTERISED BY ANOREXIA ANGULAR WITH FLUPENTIXOL AND MELITRACEN TREATMENT}

doi:10.1136/heartjnl-2012-302920aa.1

Xie Dong-yang, Cao Zuo-feng. Department of Cardiology, Affiliated Hospital of Gannan Medical College, JiangXi, GanZhou

Objectives 1. Clinical Data: The patient, female, 68 years old, came to our hospital with symptoms of anorexia, angular, lack of power with depression and fidgety for 6 months. No abnormalities were observed by electrocardiogram (ECG), chest x-ray, abdominal ultrasound and gastroscopy examination. Blood analysis showed slightly microcytic anaemia and bone marrow puncture examination showed iron-deficiency anaemia. It was lack of efficacy after taking motilium, lactic acid bacteria bacteriocin piece, JinShuangOi and the traditional Chinese medicine for spleen and stomach health. Physical examination: Blood pressure was 128/80 mm Hg, breathing was 13 times/ min, and she had sluggishness. There were scaphoid abdomen, no hepatosplenomegaly, no tenderness and normal peristaltic sound. Liver and kidney function, blood glucose, lipids and electrolytes were normal. Tumour antigen was negative. The 64-slice CT of head, chest and abdomen were normal. The psychiatrists gave psychological evaluation with anxiety scale (HAMA) and depression scale (HAMA17) after consultation. HAMA-17 scale was 28 points and HAMA scale score was 20 points, which be interpreted as moderate depression and mild anxiety. After that, the patient was added flupentixol and melitracen one tablet every morning in addition to conventional iron supplement and vitamin. The mental state, mood and sleep of patient were better than before the next day, the patient feel hungry, and appetite increased significantly. The patient continued to taking iron supplements, vitamin and flupentixol and melitracen after discharge. Follow-up after 1 month showed that the spirit, appetite, sleep, emotional and physical strength of the patient were normal, and weight gain $5 \mathrm{~kg}$. Follow-up after 2 months showed that the patient felt better, and weight gain $4 \mathrm{~kg}$ again. Then the patient stopped taking iron supplements and vitamin, and continued to taking flupentixol and melitracen (one tablet every morning). The patient felt well after half year.

Methods 2. Discussion: With the increase of competition and pressure, the rate of mental sickness is getting higher and higher, which becomes the most important healthy burden around the world.

Results This was an old female patient with anorexia and weightloss when being treated in department of gastroenterology.

Conclusions In summary: In the diagnosis and treatment of physical disease, the clinician should strive to have the habit of concern in patients with emotional and psychological state, asking whether the patient often feel depressed or even painful. If the answer is yes or suspicious, the clinician should ask patients about their feelings and subjective feeling, pay attention to the patient's sleep if they have difficulty in falling asleep, have more dreams, be easy to wake up or wake up early which is often the characteristic performance of depression. The clinicians should take the initiative to ask patients if they have the negative thoughts and suicidal thoughts, which this is very important to the diagnosis and treatment of depression. 